\title{
Association Between Social Media Use and the Acceptance of Covid-19 Vaccination Among the General Population in Saudi Arabia - A Cross- sectional Study
}

Sahar S. Othman ( $\square$ Ahbothman7@kau.edu.sa )

King Abdulaziz University

Abeer Alsuwaidi

King Abdulaziz University

Rafal Aseel

King Abdulaziz University

Reema Alotaibi

King Abdulaziz University

Reem Bablgoom

King Abdulaziz University

Ghadeer Alsulami

King Abdulaziz University

Razan Alharbi

King Abdulaziz University

Ranya Ghamri

King Abdulaziz University

\section{Research Article}

Keywords: vaccine hesitancy, vaccine acceptance, COVID-19, social media

Posted Date: November 30th, 2021

DOI: https://doi.org/10.21203/rs.3.rs-1076790/v1

License: (9) This work is licensed under a Creative Commons Attribution 4.0 International License.

Read Full License

Version of Record: A version of this preprint was published at BMC Public Health on February 21st, 2022.

See the published version at https://doi.org/10.1186/s12889-022-12757-1. 


\section{Abstract}

\section{Background}

The 2019 Coronavirus Disease Pandemic (COVID-19) is a worldwide global epidemic. Although acceptance of COVID-19 vaccination will be a critical step in combating the pandemic, achieving high uptake will be difficult, and potentially made more difficult by social media misinformation. This study aimed to examine the association between social media use and acceptance of receiving COVID-19 vaccine among the general population in Saudi Arabia.

Methodology

A cross-sectional study was conducted from June 17 to June 19, 2021 among 504 participants of the general population in Saudi Arabia. The data were collected using a three-part online questionnaire (sociodemographic characteristics, medical and vaccination history, pattern of social media use).

Results

Among 504 participants who completed the survey, 477 participants were acceptant of the vaccine and 27 were non-accepting. A total of 335 individuals had already received the vaccine, 142 were willing to receive the vaccine and 27 were unwilling. One hundred and thirty participants denied using social media for COVID-19 news. Four factors were found to be significant in influencing vaccine acceptance in univariate analysis: having a chronic condition, believing that infertility is a side effect of the COVID-19 vaccine, being concerned about a serious side effect from the vaccine and basing the decision to be vaccinated on social media information. Two of these factors fell away on multivariate analysis: basing the decision on social media information and a belief that vaccination causes infertility, suggesting that the associations are dependent on other factors.

Conclusion

In conclusion, there was no significant independent relationship between social media usage and people's willingness to receive a COVID-19 vaccination.

\section{Background}

The coronavirus disease of 2019 (COVID-19) pandemic is a global public health threat. This unexpected event has caused upheaval all around the world (1). Governments from all countries have had to act fast to prevent further spread of the virus and thus have implemented preventive measures including restrictions to travel, school closures, social distancing and the mandatory wearing of face masks $(2,3)$. It is well recognized, however, that such precautions are insufficient to contain the extension of this viral wave (1). As a result, vaccine makers have competed to produce vaccines that may potentially curb the spread of COVID-19 and possibly eradicate it $(4,5)$. 
In many countries, including the Kingdom of Saudi Arabia, COVID-19 vaccines are now available (1). Nevertheless, many people have concerns about receiving vaccines released for emergency use (6).

Attitudes towards COVID-19 vaccinations and their influencing factors have been explored in several studies (7-9). In China, Fu et al. (8) conducted a study that compared the attitudes of the general public and healthcare workers towards COVID-19 vaccination and found that the latter had a higher level of acceptance (8). Another study conducted by Thunström et al. (8) in the United States found that only $20 \%$ of people would refuse COVID-19 vaccination (8). In Pakistan, Shah et al. (9) concluded that vaccine hesitancy and rejection were mostly due to religious objection to immunization, security concerns and lack of confidence in the government (9). More recently, in October 2020, Adebisi et al. (10) studied acceptance of COVID-19 vaccination among social media users in Nigeria. The authors concluded that that most of the respondents were willing to receive COVID-19 vaccination when it became available. The main reasons for not accepting vaccination were the lack of reliability of clinical trials and the belief that the immune system is sufficient to resist the virus (10). In the United States, COVID-19 vaccine hesitancy has been associated with younger age ( $<60$ years old), lower levels of education, lower household income, rural residence and lack of health insurance (11-13).

On a local level, a few studies have examined vaccine acceptance and its determinants in Saudi Arabia (14-16). Findings have shown high hesitancy and low acceptance rates among the Saudi population (15, 16), a potential threat to public health. In the earliest of these studies, Al-Mohaithef et al. (14) concluded that attitudes towards vaccination are context specific and differ according to sociodemographics, culture and geography.

Among the factors previously found to influence the acceptance of vaccination is exposure to vaccinerelated misinformation (17), which is largely exchanged in social media (18-20). Social media networks are web-based applications that allow users to rapidly communicate ideas, thoughts and information with a large audience (21-23). Social media users present their content as a mixture of evidence, facts and personal opinions that have not been scientifically validated $(23,24)$. Furthermore, users can anonymously express their personal opinions $(24,25)$. As a result, vaccine-related misinformation is often disseminated on social media (18-20). Research has shown that exposure to such content has a negative impact on public opinion, leading to vaccine reluctance and hesitancy $(26,27)$, and this effect has been demonstrated with COVID-19 vaccine acceptance (17). Loomba et al. (17) examined the effect of exposure to COVID-19 vaccine misinformation on vaccine intent in the United Kingdom and the United States. The authors concluded that misinformation lowers vaccination intent, whereas accurate information does not (17). In the Middle East, Sallam et al. (28) conducted a study on vaccine apprehension and conspiracy ideas among Jordanians and Kuwaitis, with a small number of participants from other Arab nations, including Saudi Arabia. The authors found that using social media as a primary source of information about COVID-19 vaccination was linked to vaccine apprehension (28). This finding requires attention and further exploration to help policy makers and guide intervention programs. 
To our knowledge, no research has been published that has studied the influence of social media on the acceptance of COVID-19 vaccination among the Saudi population. This study aims to examine the association between social media use and the acceptance of COVID-19 vaccination among the general population in Saudi Arabia. We hypothesize that social media use is associated with vaccine refusal.

\section{Study objectives}

The study objectives were:

1. To estimate the rate of willingness to receive COVID-19 vaccination among the general population in Saudi Arabia as of June 2021.

2. To explore factors associated with acceptance of COVID-19 vaccination.

3. To examine the association between social media use and acceptance of COVID-19 vaccination among the general population in Saudi Arabia.

\section{Materials And Methods}

\section{Study design and data collection}

This cross-sectional analytic study used data from a survey that was conducted on the acceptability of (willingness to receive) COVID-19 vaccination among the general population in Saudi Arabia from June 17 to June 19, 2021.

The survey was constructed by the investigators after reviewing the relevant literature. Most of the questions were adopted from previous surveys of similar studies: one study on the acceptability of COVID-19 vaccination (1) and a second one that assessed COVID-19-related fake news on social media (29). Both sources had open permission to reproduce the research material $(1,29)$. The questions were then reviewed independently by two consultants ( $S O$ and $R G$ ). The survey questions were originally in English. AS, RB, GS and RO translated the questions into Arabic, and RA and RH translated them back to English to ensure that the translation was accurate and preserved the meaning of each question. The survey included questions on demographic data, medical and vaccination history, pattern of social media use, willingness to be vaccinated and reasons for refusal (if applicable). The survey was then distributed to the general population online (in both English and Arabic), using Google Forms through various social media platforms. An online approach was used to avoid physical contact during the pandemic.

As the COVID-19 vaccine was approved by the Ministry of Health during the study period for administration to adults (18 years or older), our target population was adults 18 years or older who live in Saudi Arabia. Exclusion criteria were individuals less than 18 years old, and those who have a contraindication to receiving COVID-19 vaccination (e.g., allergy to vaccine components). 
Prior to the beginning of the survey, online informed consent was obtained from the participants. This included clear information about the study objectives and the target population (eligibility to participate). Participants were clearly informed that the answers they provided would be anonymous and confidential. The informed consent provided two options: 'yes' for those who agreed to volunteer and participate in the study and 'no' for those who did not wish to participate. Only those who consented and selected 'yes' were taken to the questionnaire page to complete the survey.

The sample size was calculated by using the single proportion equation in the Raosoft software package (10). Based on the assumption that the rate of COVID-19 vaccine acceptance is $50 \%$, and a margin of error of $5 \%$ at the $95 \%$ confidence level, the required sample size was 385 . However, we received responses from 504 participants during the study period.

\section{Statistical analysis}

The primary outcome variable for this study was acceptance of receiving COVID-19 vaccination. Acceptance was measured by response to the following survey question: 'Are you willing to take the COVID-19 vaccine?'. Acceptance was defined by indicating 'yes' for the question, or by selecting the answer 'I have already taken the vaccine'. Respondents who responded with 'no', i.e., they were unwilling to be vaccinated, were asked to indicate the main reason for their unwillingness to receive vaccination.

Other information obtained from the survey was collected as categorical data. This included sociodemographic characteristics such as age, gender, marital status, the region of residency, monthly income, education level, and whether the respondent was working in the 'front line' in terms of potential exposure to COVID-19. Information on medical and vaccination history, including previous refusal of vaccines and receipt of influenza vaccine in the past was also collected. Finally, information on patterns of social media use was collected, including the use of social media for COVID-19-related news and updates and the preferred social media platform.

Statistical analysis was conducted using IBM SPSS version 20.0 software. Descriptive statistics were presented as frequencies and percentages for the whole sample, and for the two groups of our primary outcome - those acceptant of COVID-19 vaccination and those who were not. The two groups were compared using chi-square test.

The association of each predictor with the outcome ('acceptance' vs 'non-acceptance' of COVID-19 vaccination) was further tested by conducting univariate binomial regression for each variable. The predictor variables that showed a significant association $(p<0.05)$ with the outcome in the univariate analyses and those with a near significant association $(p<0.1)$ were entered into a multivariable binary logistic regression model. The level $p<0.05$ was used as the cut-off value for significance.

\section{Ethical considerations}

This study was designed and conducted in compliance with the ethical principles established by the National Committee of Bio and Medical Ethics at King Abdulaziz City for Science and Technology. Ethical 
approval was obtained from the Biomedical Research Ethics Committee, Faculty of Medicine, King Abdulaziz University (Reference number 334-21).

Table 1. Frequency distribution of participants' characteristics according to their acceptance of receiving COVID-19 vaccine $(\mathrm{N}=504)$ 
Acceptance of COVID-19 vaccine

Variable

Yes (willing to receive, or have already received the vaccine)

477 (94.6\%)
No (not willing to receive COVID-19 vaccine)

$27(5.4 \%)$

Total

\section{Age (years)}

$18-29$

30-39

40-49

$50+$
$275(57.7 \%)$

$84(17.6 \%)$

$68(14.3 \%)$

$50(10.4 \%)$
$14(51.9 \%)$

$4(14.8 \%)$

$5(18.5 \%)$

$4(14.8 \%)$
289 $(57.3 \%)$

88

(17.5\%)

73 (14.5\%)

54

$(10.7 \%)$

\section{Gender}

Female

Male

349 (73.2\%)

$128(26.8 \%)$

$19(70.4 \%)$

368

(73\%)

$8(29.6 \%)$

\section{Nationality}

Saudi

454 (95.2\%)

$26(96.3 \%)$

$1(3.7 \%)$

480

$23(4.8 \%)$

$(95.2 \%)$

24

$(4.8 \%)$

\section{Marital status}

Unmarried

$240(50.3 \%)$

$11(40.7 \%)$

251

Married

$216(45.3 \%)$

$15(55.6 \%)$

Divorced or widowed

$21(4.4 \%)$

$1(3.7 \%)$

\section{Residency area}

Southern region

$20(4.2 \%)$

$3(11.1 \%)$

23

Eastern region

0 (0\%) 


\begin{tabular}{|c|c|c|c|}
\hline Northern region & $7(1.5 \%)$ & $0(0 \%)$ & $\begin{array}{l}54 \\
(10.7 \%)\end{array}$ \\
\hline Western region & $326(68.3 \%)$ & $19(70.4 \%)$ & \\
\hline \multirow[t]{3}{*}{ Central region } & 70 (14.7\%) & $5(18.5 \%)$ & $(1.4 \%)$ \\
\hline & & & $\begin{array}{l}345 \\
(68.4 \%)\end{array}$ \\
\hline & & & $\begin{array}{l}75 \\
(14.9 \%)\end{array}$ \\
\hline
\end{tabular}

\section{Highest level of education}

$\begin{array}{llll}\text { Less than a high school diploma } & 11(2.3 \%) & 0(0 \%) & 11 \\ \text { Bachelor } & 274(57.4 \%) & 13(48.1 \%) & 2.2 \%) \\ \text { College diploma } & 27(5.7 \%) & 1(3.7 \%) & (56.9 \%) \\ \text { High School certificate } & 134(28.1 \%) & 12(44.4 \%) & 287 \\ \text { Master's/PhD } & 31(6.5 \%) & 1(3.7 \%) & (5.6 \%) \\ & & & (29 \%) \\ & & & 32 \\ & & (6.3)\end{array}$

\section{Degree in healthcare field}

No

Yes
$333(69.8 \%)$

$144(30.2 \%)$
$18(66.7 \%)$

$9(33.3 \%)$
351

(69.6\%)

153

(30.4\%)

\section{Frontline healthcare worker}

No

$423(88.7 \%)$

$22(81.5 \%)$

445

$54(11.3 \%)$

$5(18.5 \%)$

(88.3\%)
59

(11.7\%)

\section{Monthly income}

Less than SR 4000

$224(47 \%)$

Between SR 4000 - SR 10,000

$140(29.3 \%)$

$13(48.1 \%)$

$7(26 \%)$

$86(18 \%)$

$6(22.2 \%)$

$27(5.7 \%)$

$1(3.7 \%)$
237

(47\%)

147

(29.2\%)

92

(18.2\%) 


$\begin{array}{llll}\text { No } & 403(84.5 \%) & 18(66.7 \%) & 421 \\ \text { Yes } & 74(15.5 \%) & 9(33.3 \%) & (83.5 \%) \\ & & & 83- \\ & & & (16.5 \%)\end{array}$

\section{Received the seasonal Influenza}

vaccine in the past

No

yes
$204(42.8 \%)$

$273(57.2 \%)$
$13(48.1 \%)$

$14(51.9 \%)$
217

$(43.1 \%)$

287

$(56.9 \%)$

\section{Refused vaccination in the past}

No

$405(84.9 \%)$

$72(15.1 \%)$

$25(92.6 \%)$

$2(7.4 \%)$

\section{0}

$(85.3 \%)$

74

$(14.7 \%)$

Infected with COVID-19

No

$371(77.8 \%)$

$106(22.2 \%)$
$22(81.5 \%)$

$5(18.5 \%)$

\section{3}

(78\%)

111 (22\%)

\section{Family, friends or co-workers}

infected with COVID

No

Yes
$63(13.2 \%)$

$414(86.8 \%)$
$3(11.1 \%)$

$24(88.9 \%)$
66

(13.1\%)

438

$(86.9 \%)$

\section{Knowing someone passed away} due to COVID-19

No

$190(39.8 \%)$

$287(60.2 \%)$
$12(44.4 \%)$

$15(55.6 \%)$
202 $(40.1 \%)$

302 (59.9\%)

\section{Perceived risk of getting infected} with COVID-19

Low

Fair

High

$202(42.3 \%)$

$238(49.9 \%)$

$37(7.8 \%)$
$10(37 \%)$

$15(55.6 \%)$

$2(7.4 \%)$
212

$(42.1 \%)$ 253 $(50.2 \%)$ 


\section{Social media use for COVID-19} updates and news

No

Yes

$124(26 \%)$

$6(22.2 \%)$

130

$353(74 \%)$

$21(77.8 \%)$

\section{Preferred media platform for} COVID-19 news and updates

Twitter

No

Yes

178 (37.3\%)

$10(37 \%)$

188

Instagram

$299(62.7 \%)$

$17(63 \%)$

No

$395(82.8 \%)$

20 (74.1\%)

Yes

WhatsApp

82 (17.2\%)

7 (25.9\%)

No

Yes

376 (78.8\%)

17 (63\%)

101 (21.2\%)

$10(37 \%)$

Others

No

454 (95.2\%)

27 (100\%)

Yes

$23(4.8 \%)$

0 (0\%)

Side effects of the COVID-19

vaccine

Infertility.

No

Yes

Thrombosis

$432(90.6 \%)$

$45(9.4 \%)$
$20(74.1 \%)$

$7(25.9 \%)$
452

$(89.7 \%)$

52

(10.3\%) 


\begin{tabular}{|c|c|c|c|}
\hline No & 257 (53.9\%) & $13(48.1 \%)$ & \\
\hline Yes & $220(46.1 \%)$ & $14(51.9 \%)$ & $\begin{array}{l}270 \\
(53.6 \%)\end{array}$ \\
\hline \multicolumn{3}{|l|}{$\underline{\text { Sudden death }}$} & \multirow{3}{*}{$\begin{array}{l}234 \\
(46.4 \%)\end{array}$} \\
\hline No & 397 (83.2\%) & $19(70.4 \%)$ & \\
\hline Yes & $80(16.8 \%)$ & $8(29.6 \%)$ & \\
\hline \multicolumn{3}{|l|}{ Genetic changes } & \multirow[t]{2}{*}{$\begin{array}{l}416 \\
(82.5 \%)\end{array}$} \\
\hline No & 407 (85.3\%) & $21(77.8 \%)$ & \\
\hline Yes & 70 (14.7\%) & $6(22.2 \%)$ & $(17.5 \%)$ \\
\hline \multicolumn{4}{|l|}{ Others } \\
\hline No & $256(53.7 \%)$ & $19(70.4 \%)$ & $\begin{array}{l}428 \\
(84.9 \%)\end{array}$ \\
\hline \multirow[t]{3}{*}{ Yes } & \multirow[t]{3}{*}{$221(46.3 \%)$} & \multirow[t]{3}{*}{8 (29.6\%) } & $\begin{array}{l}76 \\
(15.1 \%)\end{array}$ \\
\hline & & & $\begin{array}{l}275 \\
(54.6 \%)\end{array}$ \\
\hline & & & $\begin{array}{l}229 \\
(45.4 \%)\end{array}$ \\
\hline \multicolumn{4}{|c|}{$\begin{array}{l}\text { Concerned about having a } \\
\text { serious side effect from COVID- } \\
19 \text { vaccine }\end{array}$} \\
\hline \multicolumn{4}{|l|}{ Not concerned at all } \\
\hline somewhat concerned & $266(\% 55.8)$ & $5(18.5 \%)$ & \multirow{2}{*}{$\begin{array}{l}271 \\
(53.8 \%)\end{array}$} \\
\hline \multirow[t]{3}{*}{ very concerned } & $203(42.5 \%)$ & $13(48.1 \%)$ & \\
\hline & $8(1.7 \%)$ & $9(33.3 \%)$ & $\begin{array}{l}216 \\
(42.8 \%)\end{array}$ \\
\hline & & & $\begin{array}{l}17 \\
(3.4 \%)\end{array}$ \\
\hline \multicolumn{4}{|c|}{$\begin{array}{l}\text { Decision to vaccinate is based on } \\
\text { information from social media }\end{array}$} \\
\hline \multirow[b]{2}{*}{ To some extent } & & & \multirow{2}{*}{$\begin{array}{l}238 \\
(47.2 \%)\end{array}$} \\
\hline & $231(48.4 \%)$ & $/(25.9 \%)$ & \\
\hline \multirow[t]{3}{*}{ Yes } & $145(30 \%)$ & $8(29.1 \%)$ & \multirow{2}{*}{$\begin{array}{l}151 \\
(30 \%)\end{array}$} \\
\hline & $103(21.6 \%)$ & $12(44.4 \%)$ & \\
\hline & & & $\begin{array}{l}115 \\
(22.8 \%)\end{array}$ \\
\hline
\end{tabular}




\section{Results}

As shown in Table 1, the majority of the 504 participants who completed the online survey were Saudis $(95.2 \%)$, females $(73 \%)$ and residents of the western region (68.5\%). More than half of the participants $(57.3 \%)$ were from the younger age group, i.e., between 18 and 29 years old, and were educated to Bachelor's degree (56.9\%). Among the participants, $30.4 \%$ had a degree in health care, and $11.7 \%$ were frontline healthcare workers. The monthly income for most of the participants (47\%) was $<4000$ Saudi Riyals. The rest of the data on medical and vaccination history is shown in Table 1.

Regarding social media use for COVID-19 news and updates, 374 participants $(74.2 \%)$ responded 'yes', whereas the rest $(n=130,25.8 \%)$ denied using social media for this purpose. Of those who used social media, the majority $(71.5 \%)$ reported that they strictly follow official announcements from health organizations (such as the Ministry of Health or World Health Organization [WHO]), whereas the remaining $28.5 \%$ indicated that they follow any new information available on social media, including expert opinions and personal experiences.

Among the 504 individuals who completed the survey during the study period, 335 (66.5\%) had already received COVID-19 vaccination, $142(28.2 \%)$ indicated that they are willing to receive the vaccine, and 27 (5.4\%) indicated that they are not willing to receive the vaccine. In terms of the study's primary outcome of acceptance of COVID-19 vaccination, 477 participants (94.6\%) were acceptant of the vaccine, whereas only $27(5.4 \%)$ of our sample were non-acceptant (Figure 1). The demographic characteristics, as well as the medical and vaccination information, are presented for both outcome groups in Table 1.

The 27 participants who responded as not willing to receive the COVID-19 vaccine were further asked about the reasons for refusal. The most common reason was fear of side effects (81.5\%), followed lack of long-term safety data (74.1\%). Figure 2 shows the reasons for COVID-19 vaccine refusal.

To determine predictors that are associated with the study outcome of acceptability of COVID-19 vaccine, each of the variables was first tested independently using univariant binary regression. Of all the study variables, only four were significant: having chronic conditions $(O R=0.367, p=0.0019)$, believing that infertility is a COVID-19 vaccine side effect $(O R=0.298, p=0.009)$, concern about a serious side effect from the vaccine (somewhat concerned: $O R=0.294, p=0.022$, very concerned: $O R=0.017, p<0.0001$ ) and decision to be vaccinated based on social media $(O R=0.260, p=0.006)$. The results of the univariant binary regression are shown in Table 2 . Only the variables that tested as significant $(p<0.05)$ or near significant $(p<0.1)$ are shown. The rest of the variables did not show a significant association with the outcome when tested for single effect. These results were consistent with the chi-square test results. 
Table 2

Univariate binary regression estimates of factors associated with acceptance of

\begin{tabular}{|c|c|c|c|}
\hline \multirow[t]{3}{*}{ Variable } & \multicolumn{2}{|c|}{ Acceptance of COVID-19 vaccine } & \multirow{3}{*}{$\begin{array}{l}\text { Total } \\
504 \\
(100 \%)\end{array}$} \\
\hline & $\begin{array}{l}\text { Yes (willing to receive, or } \\
\text { have already received the } \\
\text { vaccine) }\end{array}$ & $\begin{array}{l}\text { No (not willing to } \\
\text { receive COVID-19 } \\
\text { vaccine) }\end{array}$ & \\
\hline & 477 (94.6\%) & 27 (5.4\%) & \\
\hline Age (years) & $275(57.7 \%)$ & $14(51.9 \%)$ & \multirow{2}{*}{$\begin{array}{l}289 \\
(57.3 \%)\end{array}$} \\
\hline $18-29$ & $84(17.6 \%)$ & $4(14.8 \%)$ & \\
\hline $30-39$ & $68(14.3 \%)$ & $5(18.5 \%)$ & $\begin{array}{l}88 \\
(17.5 \%)\end{array}$ \\
\hline $40-49$ & $50(10.4 \%)$ & $4(14.8 \%)$ & $\begin{array}{l}73 \\
(14.5 \%)\end{array}$ \\
\hline $50+$ & & & $\begin{array}{l}54 \\
(10.7 \%)\end{array}$ \\
\hline Gender & $349(73.2 \%)$ & $19(70.4 \%)$ & \multirow{2}{*}{$\begin{array}{l}368 \\
(73 \%)\end{array}$} \\
\hline Female & \multirow[t]{2}{*}{$128(26.8 \%)$} & $8(29.6 \%)$ & \\
\hline Male & & & $\begin{array}{l}136 \\
(27 \%)\end{array}$ \\
\hline Nationality & $454(95.2 \%)$ & $26(96.3 \%)$ & \multirow{2}{*}{$\begin{array}{l}480 \\
(95.2 \%)\end{array}$} \\
\hline Saudi & \multirow[t]{2}{*}{$23(4.8 \%)$} & $1(3.7 \%)$ & \\
\hline Non-Saudi & & & $\begin{array}{l}24 \\
(4.8 \%)\end{array}$ \\
\hline Marital status & $240(50.3 \%)$ & $11(40.7 \%)$ & \multirow{2}{*}{$\begin{array}{l}251 \\
(49.8 \%)\end{array}$} \\
\hline Unmarried & $216(45.3 \%)$ & $15(55.6 \%)$ & \\
\hline Married & $21(4.4 \%)$ & $1(3.7 \%)$ & $\begin{array}{l}231 \\
(45.8 \%)\end{array}$ \\
\hline Divorced or widowed & & & $\begin{array}{l}22 \\
(4.4 \%)\end{array}$ \\
\hline
\end{tabular}




\begin{tabular}{|c|c|c|c|}
\hline \multirow[t]{3}{*}{ Variable } & \multicolumn{2}{|c|}{ Acceptance of COVID-19 vaccine } & \multirow{3}{*}{$\begin{array}{l}\text { Total } \\
504 \\
(100 \%)\end{array}$} \\
\hline & $\begin{array}{l}\text { Yes (willing to receive, or } \\
\text { have already received the } \\
\text { vaccine) }\end{array}$ & $\begin{array}{l}\text { No (not willing to } \\
\text { receive COVID-19 } \\
\text { vaccine) }\end{array}$ & \\
\hline & $477(94.6 \%)$ & $27(5.4 \%)$ & \\
\hline \multirow{7}{*}{$\begin{array}{l}\text { Residency area } \\
\text { Southern region }\end{array}$} & $20(4.2 \%)$ & $3(11.1 \%)$ & \multirow{2}{*}{$\begin{array}{l}23 \\
(4.6 \%)\end{array}$} \\
\hline & $54(11.3 \%)$ & $0(0 \%)$ & \\
\hline & $7(1.5 \%)$ & $0(0 \%)$ & $\begin{array}{l}54 \\
(10.7 \%)\end{array}$ \\
\hline & $326(68.3 \%)$ & $19(70.4 \%)$ & \multirow{2}{*}{$\begin{array}{l}7 \\
(1.4 \%)\end{array}$} \\
\hline & \multirow[t]{3}{*}{$70(14.7 \%)$} & $5(18.5 \%)$ & \\
\hline & & & $\begin{array}{l}345 \\
(68.4 \%)\end{array}$ \\
\hline & & & $\begin{array}{l}75 \\
(14.9 \%)\end{array}$ \\
\hline \multirow{3}{*}{$\begin{array}{l}\text { Highest level of education } \\
\text { Less than a high school diploma } \\
\text { Bachelor }\end{array}$} & $11(2.3 \%)$ & $0(0 \%)$ & \multirow{2}{*}{$\begin{array}{l}11 \\
(2.2 \%)\end{array}$} \\
\hline & $274(57.4 \%)$ & $13(48.1 \%)$ & \\
\hline & 27 (5.7\%) & $1(3.7 \%)$ & $\begin{array}{l}287 \\
(56.9 \%)\end{array}$ \\
\hline College diploma & 134 (28.1\%) & $12(44.4 \%)$ & $\begin{array}{l}28 \\
(5.6 \%)\end{array}$ \\
\hline \multirow{3}{*}{$\begin{array}{l}\text { High School certificate } \\
\text { Master's/PhD }\end{array}$} & \multirow[t]{3}{*}{$31(6.5 \%)$} & \multirow[t]{3}{*}{$1(3.7 \%)$} & \multirow{2}{*}{$\begin{array}{l}146 \\
(29 \%)\end{array}$} \\
\hline & & & \\
\hline & & & $\begin{array}{l}32 \\
(6.3)\end{array}$ \\
\hline \multirow{3}{*}{$\begin{array}{l}\text { Degree in healthcare field } \\
\text { No }\end{array}$} & $333(69.8 \%)$ & $18(66.7 \%)$ & \multirow{2}{*}{$\begin{array}{l}351 \\
(69.6 \%)\end{array}$} \\
\hline & \multirow[t]{2}{*}{$144(30.2 \%)$} & $9(33.3 \%)$ & \\
\hline & & & $\begin{array}{l}153 \\
(30.4 \%)\end{array}$ \\
\hline \multirow{3}{*}{$\begin{array}{l}\text { Frontline healthcare worker } \\
\text { No } \\
\text { yes }\end{array}$} & $423(88.7 \%)$ & $22(81.5 \%)$ & \multirow{3}{*}{$\begin{array}{l}445 \\
(88.3 \%) \\
59 \\
(11.7 \%)\end{array}$} \\
\hline & $54(11.3 \%)$ & $5(18.5 \%)$ & \\
\hline & & & \\
\hline
\end{tabular}




\begin{tabular}{|c|c|c|c|}
\hline \multirow[t]{3}{*}{ Variable } & \multicolumn{2}{|c|}{ Acceptance of COVID-19 vaccine } & \multirow{3}{*}{$\begin{array}{l}\text { Total } \\
504 \\
(100 \%)\end{array}$} \\
\hline & $\begin{array}{l}\text { Yes (willing to receive, or } \\
\text { have already received the } \\
\text { vaccine) }\end{array}$ & $\begin{array}{l}\text { No (not willing to } \\
\text { receive COVID-19 } \\
\text { vaccine) }\end{array}$ & \\
\hline & $477(94.6 \%)$ & $27(5.4 \%)$ & \\
\hline \multirow{5}{*}{$\begin{array}{l}\text { Monthly income } \\
\text { Less than SR } 4000 \\
\text { Between SR } 4000 \text { - SR 10,000 } \\
\text { Between SR 10,000- SR 20,000 } \\
\text { More than SR 20,000 }\end{array}$} & $224(47 \%)$ & $13(48.1 \%)$ & \multirow{2}{*}{$\begin{array}{l}237 \\
(47 \%)\end{array}$} \\
\hline & $140(29.3 \%)$ & $7(26 \%)$ & \\
\hline & $86(18 \%)$ & $6(22.2 \%)$ & $\begin{array}{l}147 \\
(29.2 \%)\end{array}$ \\
\hline & \multirow[t]{2}{*}{27 (5.7\%) } & \multirow[t]{2}{*}{$1(3.7 \%)$} & \multirow{2}{*}{$\begin{array}{l}92 \\
(18.2 \%) \\
28 \\
(5.6 \%)\end{array}$} \\
\hline & & & \\
\hline \multirow{3}{*}{$\begin{array}{l}\text { Chronic conditions } \\
\text { No } \\
\text { Yes }\end{array}$} & $403(84.5 \%)$ & $18(66.7 \%)$ & \multirow{3}{*}{$\begin{array}{l}421 \\
(83.5 \%) \\
83- \\
(16.5 \%)\end{array}$} \\
\hline & \multirow[t]{2}{*}{$74(15.5 \%)$} & \multirow[t]{2}{*}{$9(33.3 \%)$} & \\
\hline & & & \\
\hline \multirow{2}{*}{$\begin{array}{l}\text { Received the seasonal Influenza } \\
\text { vaccine in the past } \\
\text { No }\end{array}$} & $204(42.8 \%)$ & $13(48.1 \%)$ & \multirow{2}{*}{$\begin{array}{l}217 \\
(43.1 \%) \\
287 \\
(56.9 \%)\end{array}$} \\
\hline & $273(57.2 \%)$ & $14(51.9 \%)$ & \\
\hline Refused vaccination in the past & $405(84.9 \%)$ & $25(92.6 \%)$ & $\begin{array}{l}430 \\
(85,3 \%)\end{array}$ \\
\hline $\begin{array}{l}\text { No } \\
\text { Yes }\end{array}$ & $72(15.1 \%)$ & $2(7.4 \%)$ & $\begin{array}{l}74 \\
(14.7 \%)\end{array}$ \\
\hline Infected with COVID-19 & $371(77.8 \%)$ & $22(81.5 \%)$ & $\begin{array}{l}393 \\
(78 \%)\end{array}$ \\
\hline $\begin{array}{l}\text { No } \\
\text { Yes }\end{array}$ & $106(22.2 \%)$ & $5(18.5 \%)$ & $\begin{array}{l}111 \\
(22 \%)\end{array}$ \\
\hline $\begin{array}{l}\text { Family, friends or co-workers } \\
\text { infected with COVID }\end{array}$ & $63(13.2 \%)$ & $3(11.1 \%)$ & $\begin{array}{l}66 \\
(13.1 \%)\end{array}$ \\
\hline $\begin{array}{l}\text { No } \\
\text { Yes }\end{array}$ & 414 (86.8\%) & 24 (88.9\%) & $\begin{array}{l}438 \\
(86.9 \%)\end{array}$ \\
\hline
\end{tabular}




\begin{tabular}{|c|c|c|c|}
\hline \multirow[t]{3}{*}{ Variable } & \multicolumn{2}{|c|}{ Acceptance of COVID-19 vaccine } & \multirow{3}{*}{$\begin{array}{l}\text { Total } \\
504 \\
(100 \%)\end{array}$} \\
\hline & $\begin{array}{l}\text { Yes (willing to receive, or } \\
\text { have already received the } \\
\text { vaccine) }\end{array}$ & $\begin{array}{l}\text { No (not willing to } \\
\text { receive COVID-19 } \\
\text { vaccine) }\end{array}$ & \\
\hline & $477(94.6 \%)$ & $27(5.4 \%)$ & \\
\hline \multirow{2}{*}{$\begin{array}{l}\text { Knowing someone passed away } \\
\text { due to COVID-19 } \\
\text { No } \\
\text { yes }\end{array}$} & $190(39.8 \%)$ & $12(44.4 \%)$ & \multirow{2}{*}{$\begin{array}{l}202 \\
(40.1 \%) \\
302 \\
(59.9 \%)\end{array}$} \\
\hline & $287(60.2 \%)$ & $15(55.6 \%)$ & \\
\hline \multirow{2}{*}{$\begin{array}{l}\text { Perceived risk of getting infected } \\
\text { with COVID-19 }\end{array}$} & $202(42.3 \%)$ & $10(37 \%)$ & \multirow{2}{*}{$\begin{array}{l}212 \\
(42.1 \%)\end{array}$} \\
\hline & $238(49.9 \%)$ & $15(55.6 \%)$ & \\
\hline Low & $37(7.8 \%)$ & $2(7.4 \%)$ & $\begin{array}{l}253 \\
(50.2 \%)\end{array}$ \\
\hline High & & & $\begin{array}{l}39 \\
(7.7 \%)\end{array}$ \\
\hline $\begin{array}{l}\text { Social media use for COVID-19 } \\
\text { updates and news }\end{array}$ & $124(26 \%)$ & $6(22.2 \%)$ & $\begin{array}{l}130 \\
(25.8 \%)\end{array}$ \\
\hline $\begin{array}{l}\text { No } \\
\text { Yes }\end{array}$ & $353(74 \%)$ & $21(77.8 \%)$ & $\begin{array}{l}374 \\
(74.2 \%)\end{array}$ \\
\hline \multirow{2}{*}{$\begin{array}{l}\text { Preferred media platform for } \\
\text { COVID-19 news and updates }\end{array}$} & $178(37.3 \%)$ & $10(37 \%)$ & \multirow{2}{*}{$\begin{array}{l}188 \\
(37.3 \%)\end{array}$} \\
\hline & $299(62.7 \%)$ & $17(63 \%)$ & \\
\hline & $395(82.8 \%)$ & $20(74.1 \%)$ & $\begin{array}{l}316 \\
(62.7 \%)\end{array}$ \\
\hline \multirow{2}{*}{ Yes } & $82(17.2 \%)$ & $7(25.9 \%)$ & \multirow{2}{*}{$\begin{array}{l}415 \\
(82.3 \%)\end{array}$} \\
\hline & $376(78.8 \%)$ & $17(63 \%)$ & \\
\hline Instagram & $101(21.2 \%)$ & $10(37 \%)$ & $\begin{array}{l}89 \\
(17.7 \%)\end{array}$ \\
\hline \multirow{2}{*}{ Yes } & $454(95.2 \%)$ & $27(100 \%)$ & \multirow{2}{*}{$\begin{array}{l}393 \\
(78 \%)\end{array}$} \\
\hline & $23(4.8 \%)$ & $0(0 \%)$ & \\
\hline WhatsApp & & & $\begin{array}{l}111 \\
(22 \%)\end{array}$ \\
\hline Yes & & & $\begin{array}{l}481 \\
(95.4 \%)\end{array}$ \\
\hline \multicolumn{2}{|l|}{ Others } & & \multirow[t]{3}{*}{$\begin{array}{l}23 \\
(4.6 \%)\end{array}$} \\
\hline \multicolumn{3}{|l|}{ No } & \\
\hline \multicolumn{3}{|l|}{ Yes } & \\
\hline
\end{tabular}




\begin{tabular}{|c|c|c|c|}
\hline \multirow[t]{3}{*}{ Variable } & \multicolumn{2}{|c|}{ Acceptance of COVID-19 vaccine } & \multirow{3}{*}{$\begin{array}{l}\text { Total } \\
504 \\
(100 \%)\end{array}$} \\
\hline & $\begin{array}{l}\text { Yes (willing to receive, or } \\
\text { have already received the } \\
\text { vaccine) }\end{array}$ & $\begin{array}{l}\text { No (not willing to } \\
\text { receive COVID-19 } \\
\text { vaccine) }\end{array}$ & \\
\hline & $477(94.6 \%)$ & $27(5.4 \%)$ & \\
\hline \multirow{2}{*}{$\begin{array}{l}\text { Side effects of the COVID-19 } \\
\text { vaccine }\end{array}$} & $432(90.6 \%)$ & $20(74.1 \%)$ & \multirow{2}{*}{$\begin{array}{l}452 \\
(89.7 \%)\end{array}$} \\
\hline & $45(9.4 \%)$ & $7(25.9 \%)$ & \\
\hline Infertility & $257(53.9 \%)$ & $13(48.1 \%)$ & $\begin{array}{l}52 \\
(10.3 \%)\end{array}$ \\
\hline \multirow{2}{*}{ Yes } & $220(46.1 \%)$ & $14(51.9 \%)$ & \multirow{2}{*}{$\begin{array}{l}270 \\
(53.6 \%)\end{array}$} \\
\hline & 397 (83.2\%) & 19 (70.4\%) & \\
\hline Thrombosis & $80(16.8 \%)$ & $8(29.6 \%)$ & $\begin{array}{l}234 \\
(46.4 \%)\end{array}$ \\
\hline No & $407(85.3 \%)$ & $21(77.8 \%)$ & \multirow{2}{*}{$\begin{array}{l}416 \\
(82.5 \%)\end{array}$} \\
\hline \multirow{2}{*}{ Sudden death } & $70(14.7 \%)$ & $6(22.2 \%)$ & \\
\hline & $256(53.7 \%)$ & 19 (70.4\%) & $\begin{array}{l}88 \\
(17.5 \%)\end{array}$ \\
\hline Yes & $221(46.3 \%)$ & $8(29.6 \%)$ & $\begin{array}{l}428 \\
(84.9 \%)\end{array}$ \\
\hline \multirow{2}{*}{\multicolumn{2}{|c|}{ Genetic changes }} & & \multirow[b]{2}{*}{$\begin{array}{l}76 \\
(15.1 \%)\end{array}$} \\
\hline & & & \\
\hline \multirow{2}{*}{\multicolumn{3}{|c|}{ Yes }} & \multirow{2}{*}{$\begin{array}{l}275 \\
(54.6 \%)\end{array}$} \\
\hline & & & \\
\hline Others & & & \multirow{3}{*}{$\begin{array}{l}229 \\
(45.4 \%)\end{array}$} \\
\hline \multicolumn{3}{|l|}{ No } & \\
\hline \multicolumn{3}{|l|}{ Yes } & \\
\hline \multirow{2}{*}{$\begin{array}{l}\text { Concerned about having a } \\
\text { serious side effect from COVID- } \\
19 \text { vaccine }\end{array}$} & $266(\% 55.8)$ & $5(18.5 \%)$ & \multirow{2}{*}{$\begin{array}{l}271 \\
(53.8 \%)\end{array}$} \\
\hline & $203(42.5 \%)$ & $13(48.1 \%)$ & \\
\hline Not concerned at all & $8(1.7 \%)$ & $9(33.3 \%)$ & $\begin{array}{l}216 \\
(42.8 \%)\end{array}$ \\
\hline \multirow{2}{*}{$\begin{array}{l}\text { somewhat concerned } \\
\text { very concerned }\end{array}$} & & & \multirow[t]{2}{*}{$\begin{array}{l}17 \\
(3.4 \%)\end{array}$} \\
\hline & & & \\
\hline
\end{tabular}




\begin{tabular}{|llll|}
\hline Variable & \multicolumn{2}{l}{ Acceptance of COVID-19 vaccine } & Total \\
\cline { 2 - 3 } & $\begin{array}{l}\text { Yes (willing to receive, or } \\
\text { have already received the } \\
\text { vaccine) }\end{array}$ & $\begin{array}{l}\text { No (not willing to } \\
\text { receive COVID-19 } \\
\text { vaccine) }\end{array}$ & $\begin{array}{l}\mathbf{5 0 4} \\
\mathbf{( 1 0 0 \% )}\end{array}$ \\
\hline $\begin{array}{l}\text { Decision to vaccinate is based on } \\
\text { information from social media }\end{array}$ & $231(\mathbf{4 7 . 6 \% )}$ & $\mathbf{2 7}(\mathbf{5 . 4 \% )}$ & \\
No & $143(30 \%)$ & $7(25.9 \%)$ & 238 \\
To some extent & $103(21.6 \%)$ & $8(29.7 \%)$ & $(47.2 \%)$ \\
Yes & & $12(44.4 \%)$ & 151 \\
\hline COVID-19 vaccination. & & & $(30 \%)$ \\
\hline
\end{tabular}

Next, the seven variables that had $p<0.1$ in the univariant binary regression were entered into a multivariable logistic regression model. As shown in Table 3, two of the significant variables in the univariant regression remained significant in the multivariable model: having chronic conditions $(\mathrm{OR}=$ $0.331, p=0.036$ ) and concern about a serious side effect from vaccination (very concerned $O R=0.019$, $p<0.0001)$. The other two variables did not show a significant effect when entered into the multivariable model: believing that infertility is a COVID-19 vaccine side effect $(O R=0.0333, p=0.054)$, and vaccination decision based on social media $(\mathrm{OR}=0.356, \mathrm{p}=0.071)$. The rest of the variables in the model were all nonsignificant. The model was overall significant $(p<0.001, R 2=0.302)$. 
Table 3

Multivariate logistic regression estimates of factors associated with acceptance of

\begin{tabular}{|c|c|c|c|c|c|}
\hline \multirow[t]{2}{*}{ Predictor } & & \multirow{2}{*}{$\begin{array}{l}\text { p- } \\
\text { value }\end{array}$} & \multirow{2}{*}{$\begin{array}{c}\text { Odds } \\
\text { Ratio } \\
\text { (OR) }\end{array}$} & \multicolumn{2}{|c|}{ 95\% C.I. for OR } \\
\hline & & & & Lower & Upper \\
\hline Presence of chronic conditions & yes & 0.019 & 0.367 & 0.159 & 0.849 \\
\hline $\begin{array}{l}\text { Preferred Media platform for Coronavirus } \\
\text { News and updates: What's App }\end{array}$ & yes & 0.058 & 0.457 & 0.203 & 1.028 \\
\hline $\begin{array}{l}\text { Side effects of the COVID-19 vaccine: } \\
\text { Infertility }\end{array}$ & yes & 0.009 & 0.298 & 0.119 & 0.742 \\
\hline $\begin{array}{l}\text { Side effects of the COVID-19 vaccine: Sudden } \\
\text { death }\end{array}$ & yes & 0.093 & 0.479 & 0.202 & 1.131 \\
\hline Side effects of the COVID-19 vaccine: others & yes & 0.096 & 2.050 & 0.880 & 4.775 \\
\hline \multirow[t]{3}{*}{$\begin{array}{l}\text { Concerned about serious side effect from the } \\
\text { vaccine }\end{array}$} & $\begin{array}{l}\text { not concerned } \\
\text { at all }\end{array}$ & & Ref & & \\
\hline & $\begin{array}{l}\text { somewhat } \\
\text { concerned }\end{array}$ & 0.022 & 0.294 & 0.103 & 0.837 \\
\hline & $\begin{array}{l}\text { very } \\
\text { concerned }\end{array}$ & 0.000 & 0.017 & 0.005 & 0.061 \\
\hline \multirow{3}{*}{$\begin{array}{l}\text { Decision based on information in social } \\
\text { media }\end{array}$} & no & & Ref & & \\
\hline & $\begin{array}{l}\text { to some } \\
\text { extent }\end{array}$ & 0.246 & 0.542 & 0.192 & 1.526 \\
\hline & yes & 0.006 & 0.260 & 0.100 & 0.680 \\
\hline
\end{tabular}

\section{Discussion}

Social media plays an important role as a source of information, especially during this pandemic, during which users have sought the most up-to-date information; however, information disseminated via social media can be misleading. The goal of this study was to examine for a link between social media use and acceptance of COVID-19 vaccination in Saudi Arabia's general public.

Most of the respondents of our survey were in younger age groups with more than half of the received responses (57.3\%) from individuals between 18 and 29 years old. This response can be explained by the fact that most social media users are young. The majority were vaccinated or acceptant of COVID-19 vaccination. The percentage of the vaccinated individuals was high (66.5\%) in comparison to the acceptance rates found in a previous study conducted by Qattan et al. (1) in Saudi Arabia. This compares with findings from the US, where vaccine hesitancy rates also appear to have decreased. In an internet 
survey of approximately 3500 adults conducted by the Centers for Disease Control (CDC) in September and December 2020, the proportion of adults who reported that they were very likely or absolutely certain to receive a COVID-19 vaccine increased from $39-49 \%$, and the proportion who were unlikely to receive one decreased from 38-32\% (11). In another survey of approximately 7200 adults, rates of vaccine hesitancy decreased from 46\% in October 2020 to 35\% in March 2021 (12, 30, 31). Two reasons explain the significant decrease in rates of vaccine hesitancy. Firstly, as more time lapses following vaccine approval, people become more confident of the safety of vaccination. Secondly, vaccination is becoming mandatory in more places in Saudi Arabia, including for travel, for access to religious/worship settings, as well as schools and commercial buildings.

Reasons for unwillingness to be vaccinated, shown in Figure 2, were fear of side effects (81.5\%), insufficient length of time of testing (74.0\%), a belief that vaccination is ineffective (48.2\%) and other/personal reasons (22.2\%). This is consistent with the findings of the CDC survey, where the main reasons for reporting non-intent to receive the vaccine were concerns about its safety and side effects, and a lack of trust in the process (11).

When tested for single effect, four variables showed a significant association with vaccine decision having a chronic condition, believing that infertility is a side effect, concerns about side effects and making a decision based on social media. All those factors showed a negative effect on the outcome, i.e. making the acceptance less likely, and this result was consistent across analyses. However, deciding based on social media and fear of fertility were not significant when tested for combined effect - only concerns about serious side effects and having a chronic condition remained significantly independently associated with vaccine hesitancy.

The association between having a chronic condition and vaccine hesitancy, which was significant in both analyses, may be explained by a heightened fear of an adverse event or complication following vaccination. Concerns of serious side effects is a logical reason for vaccine rejection, and this was significant in both analyses. Fear of infertility was expressed by some participants, but was not independent of other factors.

A previous study that took place in Riyadh (32) found no significant impact of social media exposure on vaccine hesitancy; however, our findings from the single factor analysis suggest there may be some relationship between social media use and vaccine decision. Our hypothesis was rejected in the multivariate analysis, suggesting that this association is dependent on other factors. Although the internet and social media networks can contribute crucial information, they can also misrepresent the facts, causing distrust and misunderstanding. Our results suggest that healthcare organizations and governments need to provide reliable vaccination information via social media platforms, to influence vaccine hesitancy.

Our study has a number of limitations. It was an observational study conducted at one point in time, therefore no causal relationship between the factors and the outcome can be inferred. The majority of respondents were from the western area of Saudi Arabia, and the limited response from some areas 
indicates that the sample was not entirely representative. Finally, the introduction of mandatory vaccination in some contexts in Saudi Arabia potentially confounded or masked the relationships of some variables.

\section{Abbreviations}

COVID-19

The Coronavirus disease of 2019

WHO

World Health Organization

OR

Odds ratio

$\mathrm{Cl}$

Confidence interval

\section{Declarations}

\section{Ethics approval and consent to participate}

Ethical approval was obtained from the Biomedical Research Ethics Committee, Faculty of Medicine, King Abdulaziz University (Reference number 334-21). Written informed consent was obtained from each participant prior to beginning the survey.

\section{Consent for publication}

Not applicable

\section{Availability of data and materials}

The datasets used and/or analysed during the current study are available from the corresponding author on reasonable request.

\section{Competing interests}

The authors declare that they have no competing interests

\section{Funding}

Self-funded

\section{Author's contribution:}

Abeer Alsuwaidi, Reem Bablgoom, Ghadeer alsulami, Reema Alotaibi: development of study proposal and questionnaire, translation of questionnaire from English to Arabic, data collection, writing parts of the 
manuscript.

Rafal Aseel, Razan Alharbi: development of study proposal and questionnaire, translation of questionnaire from Arabic back to English, data collection, writing parts of the manuscript.

Sahar Othman: Review of proposal and questionnaire, data analysis, interpretation of results, writing parts of the manuscript and final editing.

Ranya Ghamri: Review of questionnaire, interpretation of results, final editing of the manuscript.

\section{Acknowledgements}

Not applicable

\section{References}

1 Qattan A, Alshareef N, Alsharqi O, Al Rahahleh N, Chirwa GC, Al-Hanawi MK. Acceptability of a COVID19 vaccine among healthcare workers in the Kingdom of Saudi Arabia. Front Med. 2021;8:83.

2 Al-Hanawi MK, Angawi K, Alshareef N, et al. Knowledge, attitude and practice toward COVID-19 among the public in the Kingdom of Saudi Arabia: a cross-sectional study. Front Public Health. 2020;8:217.

3 Sherman SM, Smith LE, Sim J, et al. COVID-19 vaccination intention in the UK: results from the COVID19 vaccination acceptability study (CoVAccS), a nationally representative cross-sectional survey. Hum Vaccin Immunother. 2021;17(6):1612-1621.

4 Lurie N, Saville M, Hatchett R, Halton J. Developing Covid-19 vaccines at pandemic speed. N Engl J Med. 2020;382(21):1969-1973.

5 Yang Y, Peng F, Wang R, et al. The deadly coronaviruses: The 2003 SARS pandemic and the 2020 novel coronavirus epidemic in China. J Autoimmun. 2020;109:102434.

6 Rhodes A, Hoq M, Measey MA, Danchin M. Intention to vaccinate against COVID-19 in Australia. Lancet Infect Dis. 2021;21(5):e110.

7 Thunstrom L, Ashworth M, Finnoff D, Newbold S. Hesitancy towards a COVID-19 vaccine. Ecohealth. 2021;18(1):44-60.

8 Fu C, Wei Z, Pei S, Li S, Sun X, Liu P. Acceptance and preference for COVID-19 vaccination in healthcare workers (HCWs) [preprint]. MedRxiv. 2020. https://doi.org/10.1101/2020.04.09.20060103.

9 Shah SF, Ginossar T, Weiss D. "This is a Pakhtun disease": Pakhtun health journalists' perceptions of the barriers and facilitators to polio vaccine acceptance among the high-risk Pakhtun community in Pakistan. Vaccine. 2019;37(28):3694-3703. 
10 Adebisi YA, Alaran AJ, Bolarinwa OA, Akande-Sholabi W, Lucero-Prisno DE. When it is available, will we take it? Social media users' perception of hypothetical COVID-19 vaccine in Nigeria. Pan Afr Med. 2021;38:230.

11 Nguyen KH, Srivastav A, Razzaghi H, et al. COVID-19 Vaccination Intent, Perceptions, and Reasons for Not Vaccinating Among Groups Prioritized for Early Vaccination. MMWR Morb Mortal Wkly Rep. 2021;70:217-222.

12 Szilagyi PG, Thomas K, Shah MD, et al. National Trends in the US Public's Likelihood of Getting a COVID-19 Vaccine-April 1 to December 8, 2020; JAMA, 2020;325(4):396-398.

13 Fisher KA, Bloomstone SJ, Walder J, Crawford S, Fouayzi H, Mazor KM. Attitudes Toward a Potential SARS-CoV-2 Vaccine: A Survey of U.S. Adults. Ann Intern Med. 2020;173(12):964.

14 Al-Mohaithef M, Padhi BK. Determinants of COVID-19 vaccine acceptance in Saudi Arabia: a webbased national survey. J Multidiscip Healthc. 2020;13:1657-1663.

15 Alfageeh El, Alshareef N, Angawi K, Alhazmi F, Chirwa GC. Acceptability of a COVID-19 Vaccine among the Saudi population. Vaccines. 2021;9(3):226.

16 Almaghaslah D, Alsayari A, Kandasamy G, Vasudevan R. COVID-19 Vaccine Hesitancy among Young Adults in Saudi Arabia: A Cross-Sectional Web-Based Study. Vaccines. 2021;9(4):330.

17 Loomba S, de Figueiredo A, Piatek SJ, de Graaf K, Larson HJ. Measuring the impact of exposure to COVID-19 vaccine misinformation on vaccine intent in the UK and US. Nat Hum Behav. 2021.5(3):337348.

18 Meleo-Erwin Z, Basch C, MacLean SA, Scheibner C, Cadorett V. "To each his own": Discussions of vaccine decision-making in top parenting blogs. Hum Vaccin Immunother. 2017;13(8):1895-1901.

19 Oehler RL. On Measles, Vaccination, Social Media Activism, and How to Win Back Our Role as Our Patients' Best Advocates. Clin Infect Dis. 2020;70(2):338-340.

20 Ortiz RR, Smith A, Coyne-Beasley T. A systematic literature review to examine the potential for social media to impact HPV vaccine uptake and awareness, knowledge, and attitudes about HPV and HPV vaccination. Hum Vaccin Immunother. 2019;15(7-8):1465-1475.

21 Puri N, Coomes EA, Haghbayan H, Gunaratne K. Social media and vaccine hesitancy: new updates for the era of COVID-19 and globalized infectious diseases. Hum Vaccin Immunother. 2020;16(11):1-8.

22- Betsch C, Brewer NT, Brocard P, et al. Opportunities and challenges of Web 2.0 for vaccination decisions. Vaccine. 2012;30(25):3727-3733. 
23 Wilson K, Keelan J. Social media and the empowering of opponents of medical technologies: the case of anti-vaccinationism. J Med Internet Res. 2013;15(5):e103.

24 Buller DB, Walkosz BJ, Berteletti J, et al. Insights on HPV vaccination in the United States from mothers' comments on Facebook posts in a randomized trial. Hum Vaccin Immunother. 2019;15(78):1479-1487.

25 Massey PM, Budenz A, Leader A, Fisher K, Klassen AC, Yom-Tov E. Peer Reviewed: What Drives Health Professionals to Tweet About \#HPVvaccine? Identifying Strategies for Effective Communication. Prev Chronic Dis. 2018;15:E26.

26 Betsch C, Renkewitz F, Betsch T, Ulshöfer C. The influence of vaccine-critical websites on perceiving vaccination risks. J Health Psychol. 2010;15(3):446-455.

27 Nan X, Madden K. HPV vaccine information in the blogosphere: how positive and negative blogs influence vaccine-related risk perceptions, attitudes, and behavioral intentions. Health Commun. 2012;27(8):829-836.

28 Sallam M, Dababseh D, Eid H, et al. High Rates of COVID-19 Vaccine Hesitancy and Its Association with Conspiracy Beliefs: A Study in Jordan and Kuwait among Other Arab Countries. Vaccines. 2021;9(1):42.

29 Apuke OD, Omar B. Fake news and COVID-19: modelling the predictors of fake news sharing among social media users. Telemat and Inform. 2021;56:101475.

30 Daly M, Jones A, Robinson E. Public Trust and Willingness to Vaccinate Against COVID-19 in the US From October 14, 2020, to March 29, 2021. JAMA. 2021;325(23):2397.

31 Gadoth A, Halbrook M, Martin-Blais R, et al. Cross-sectional Assessment of COVID-19 Vaccine Acceptance Among Health Care Workers in Los Angeles. Ann Intern Med. 2021;174(6):882.

32 Al-Regaiey KA, Alshamry WS, Alqarn RA, et al. Influence of social media on parents' attitudes towards vaccine administration [online ahead of print]. Hum Vaccin Immunother. 2021; Feb17:1-8.

\section{Figures}




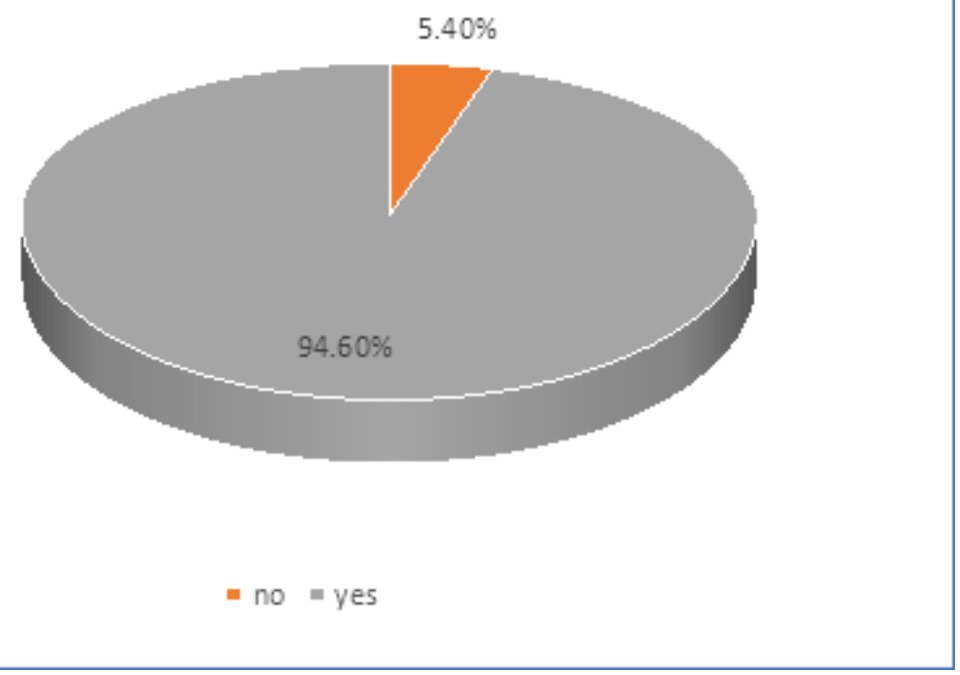

Figure 1

Acceptance of COVID-19 vaccine

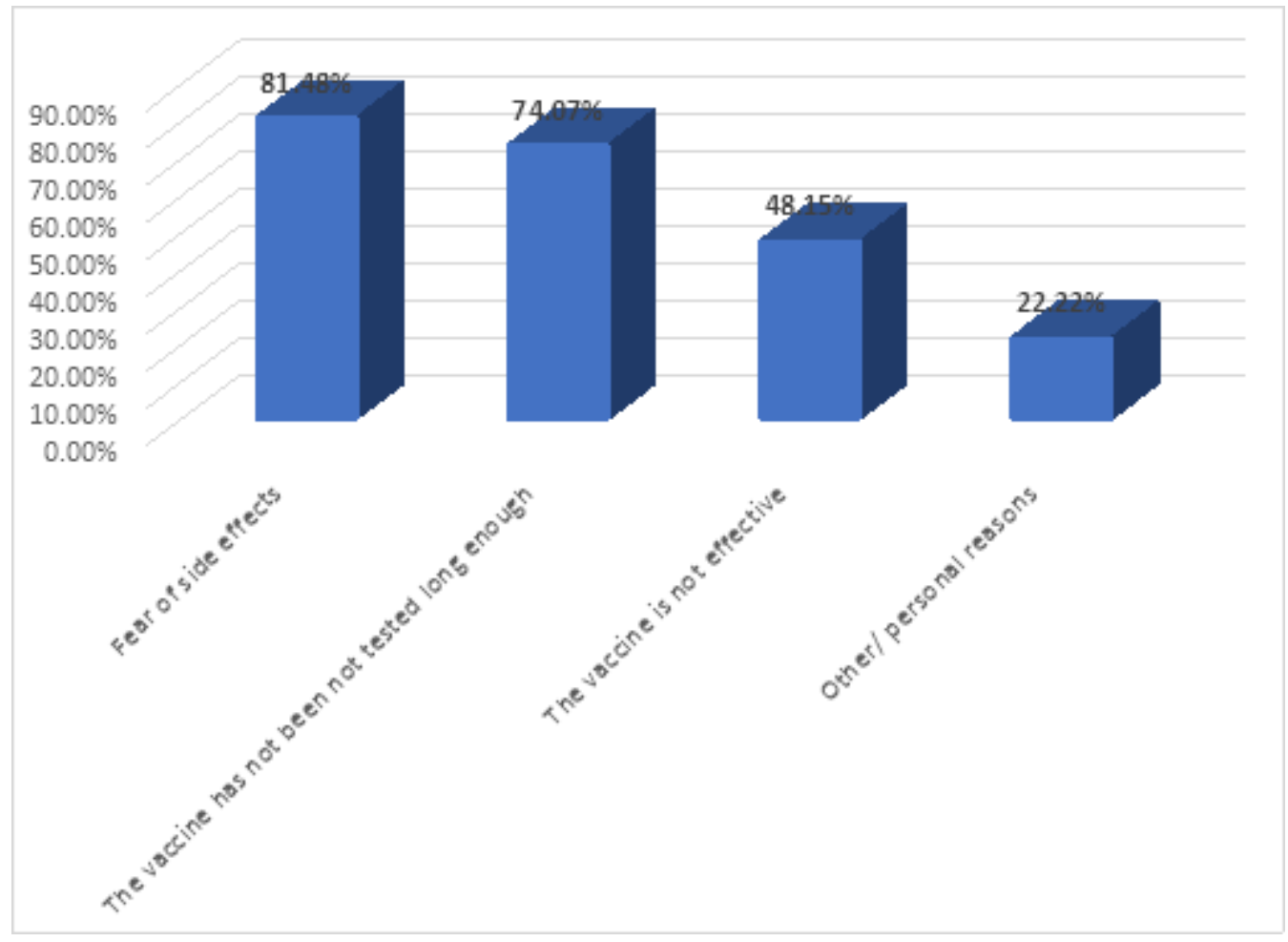

Figure 2

Reasons for refusal of COVID-19 vaccine. 